\title{
Convective core mixing: A metallicity dependence?
}

\author{
D. Cordier ${ }^{1,2}$, Y. Lebreton ${ }^{1}$, M.-J. Goupil ${ }^{1}$, T. Lejeune ${ }^{3}$, J.-P. Beaulieu ${ }^{4}$, and F. Arenou ${ }^{1}$ \\ 1 DASGAL, CNRS UMR 8632, Observatoire de Paris-Meudon, DASGAL, 92195 Meudon Principal Cedex, France \\ 2 École Nationale Supérieure de Chimie de Rennes, Campus de Beaulieu, 35700 Rennes, France \\ 3 Observatorio Astronomico, Universidade de Coimbra, Santa Clara 3040 Coimbra, Portugal \\ ${ }^{4}$ I.A.P., 98 bis boulevard Arago, 75014 Paris, France
}

Received 5 March 2002 / Accepted 6 June 2002

\begin{abstract}
The main purpose of this paper is to investigate the possible existence of a metallicity dependence of the overshooting from main sequence star turbulent cores. We focus on objects with masses in the range $\sim 2.5 M_{\odot}-\sim 25 M_{\odot}$. Evolutionary time scale ratios are compared with star number ratios on the main sequence. Star populations are synthesized using grids of evolutionary tracks computed with various overshooting amounts. Observational material is provided by the large and homogeneous photometric database of the OGLE 2 project for the Magellanic clouds. Attention is paid to the study of uncertainties: distance modulus, intergalactic and interstellar reddening, IMF slope and average binarity rate. Rotation and the chemical composition gradient are also considered. The result for the overshooting distance is $l_{\text {over }}^{\mathrm{SMC}}=0.40_{-0.06}^{+0.12} \mathrm{H}_{\mathrm{p}}\left(Z_{0}=0.004\right)$ and $l_{\mathrm{over}}^{\mathrm{LMC}}=0.10_{-0.10}^{+0.17} \mathrm{H}_{\mathrm{p}}$ $\left(Z_{0}=0.008\right)$ suggesting a possible dependence of the extent of the mixed central regions with metallicity within the considered mass range. Unfortunately it is not yet possible to fully disentangle the effects of mass and chemical composition.
\end{abstract}

Key words. convection - stars: evolution, interiors

\section{Introduction}

Extensive convective phenomena occur in the cores of main sequence stars with masses above about $1.2 M_{\odot}$ (for galactic chemical composition). In standard models, convection is crudely modeled with the well-known Mixing Length Theory of Böhm-Vitense (1958) (hereafter MLT) and the core extension is determined according to the Schwarzschild criterion. The Schwarzschild limit is the value of the radius where the buoyancy force vanishes. However, inertia of the convective elements leads to an extra mixing above the Schwarzschild limit, called "overshooting" and is usually expressed as a fraction of the pressure scale height. Several theoretical works (for a review see Zahn 1991) give arguments in favor of such additional mixing. Many laboratory experiments show evidence for overshooting (see Massaguer 1990). Although overshooting can occur below an external convective zone (see Alongi et al. 1991), this paper is exclusively concerned with core overshooting.

One of the first empirical determinations of convective core overshooting was obtained by Maeder \& Mermilliod (1981) who used a set of 34 galactic open clusters and fitted the main sequence width with an additional mixing of about 20-40\% in mass fraction. Mermilliod \& Maeder (1986) derived an overshooting amount of about $0.3 \mathrm{H}_{\mathrm{p}}$ for solar-like chemical composition and for a 9-15 $M_{\odot}$ range. Stothers \& Chin (1991) derived an overshooting amount $<0.2 \mathrm{H}_{\mathrm{p}}$ for Pop. I stars using the

Send offprint requests to: D. Cordier, e-mail: daniel.cordier@ensc-rennes.fr metal-enriched opacity tables published in Rogers \& Iglesias (1992)

During the last decade, many evolutionary model grids have been computed with an overshooting amount equal or close to $0.2 \mathrm{H}_{\mathrm{p}}$ : e.g. Charbonnel et al. (1996) or Bertelli et al. (1994). This second team (Padova group) uses a formalism (see Bressan et al. 1981) slightly different from the Geneva team one (e.g. see Schaller et al. 1992). Generally the same overshooting amount is used whatever the metallicity and mass are.

Kozhurina-Platais et al. (1997) obtained $l_{\text {over }}=0.2 \pm$ $0.05 \mathrm{H}_{\mathrm{P}}$ for the galactic cluster NGC 3680 (solar metallicity) with the isochrone technique. This method consists of fitting the cluster CMD features (particularly the turn-off position) with model isochrones. Iwamoto \& Saio (1999) compared evolutionary models with observations of three binary systems: V2291 Oph, $\alpha$ Aur and $\eta$ And ("binary system" technique). The authors adjusted either the helium content or the overshooting parameter to get a better fit to observations. The best results were obtained with a moderate overshooting amount $\left(\lesssim 0.15 \mathrm{H}_{\mathrm{p}}\right)$. For super-solar metallicity $\left(Z_{0}=0.024\right)$ Lebreton et al. (2001) derived $l_{\text {over }} \lesssim 0.2 \mathrm{H}_{\mathrm{P}}$ from the modeling of the Hyades cluster turn-off.

Maeder \& Mermilliod (1981) have suggested an overshooting increasing with mass within the studied range of 2-6 $M_{\odot}$ which is also found by Schröder et al. (1997) with a study of binary systems. According to their results, the overshooting should increase from $\lesssim 0.24 \mathrm{H}_{\mathrm{p}}$ for $2.5 M_{\odot}$ to $\lesssim 0.32 \mathrm{H}_{\mathrm{p}}$ for $6.5 M_{\odot}$. With a similar study Ribas et al. (2000) also found a mass dependence. 


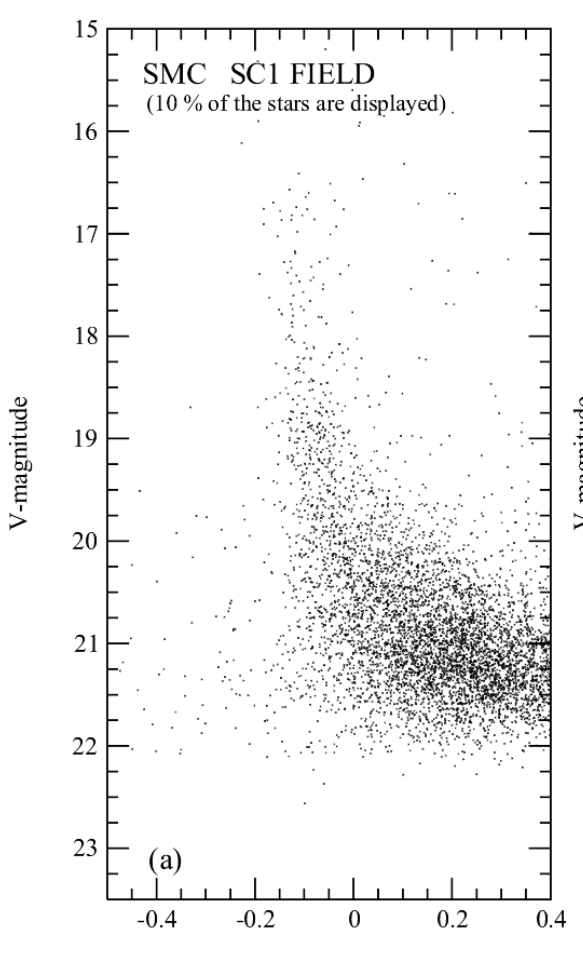

$(\mathrm{B}-\mathrm{V})$

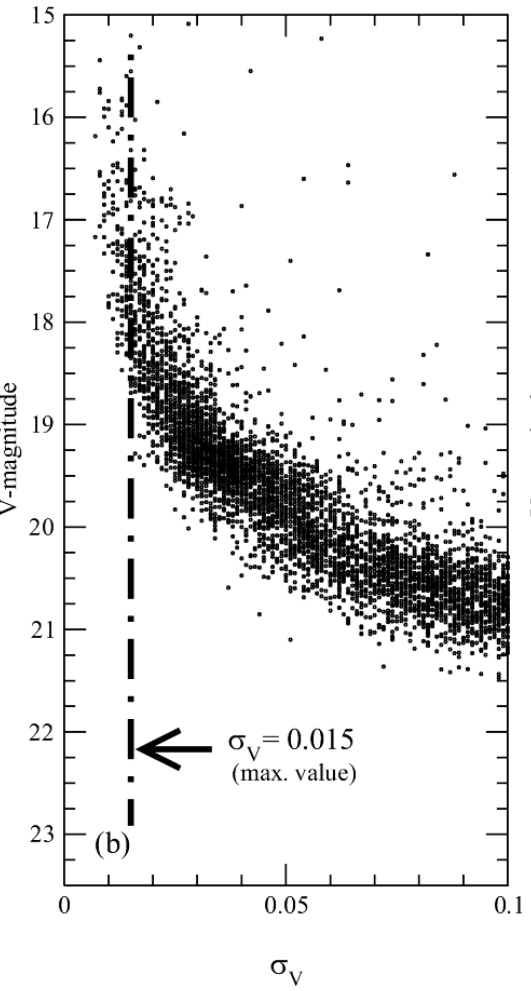

Fig. 1. a) CM-Diagram for the OGLE 2 SC 1 field, $10 \%$ of the data have been plotted for sake of clarity. b) Standard deviation of the measurements: $\sigma_{V}$ versus magnitude $V$, the dot-dashed line indicates the limit-value considered (0.015). c) The resulting CM-diagram after selection (all the fields within SMC have been plotted).

The question of a metallicity dependence must also be addressed. Ribas et al. (2000)'s results suggest a slight metallicity dependence for a stellar mass around $2.40 M_{\odot}$ (see their Table 1). The more metal poor star SZ Cen (in mass fraction: $\left.Z_{0}=0.007\right)$ is satisfactorily modelled with an overshooting distance $0.1 \mathrm{H}_{\mathrm{p}} \lesssim l_{\mathrm{ov}} \lesssim 0.2 \mathrm{H}_{\mathrm{P}}$ and objects with $Z_{0}$ ranging between 0.015 and 0.020 seem to have an overshooting around $0.2 \mathrm{H}_{\mathrm{P}}$. Keller et al. (2001) have recently explored the dependence of overshooting with metallicity by means of the isochrone technique using isochrone grids from the Padova group. Their study involves HST observations of four clusters: NGC 330 (SMC), 1818, 2004 and 2001 (LMC). Keller et al. (2001) find the best fit (with respect to age and overshooting) for an overshooting amount which is equivalent to $l_{\text {over }}=0.31 \pm 0.11 \mathrm{H}_{\mathrm{p}}$ in the Geneva formalism $\left(l_{\mathrm{over}}^{\text {Padova }}=\right.$ $2 \times l_{\text {over }}^{\text {Geneva }}$.

In this paper, we carry out an independent study of a possible metallicity dependence of overshooting with a technique which differs from the "binary system" (Ribas et al. 2000; Andersen 1991) and "isochrone" techniques. Our method is based on star-count ratios, with comparisons between observational material and synthetic population results in colormagnitude (CMD) diagrams. We are then led to discuss several points: particularly distance modulus, reddening and binarity rate. If the dependence of overshooting on metallicity (or mass) was thereby to be firmly assessed, it would then be a challenge to understand its physical origin.

We are concerned with a metallicity range relevant to the Magellanic Clouds and take advantage of the homogeneous
OGLE 2 data, which provide color magnitude diagrams for $\sim 2 \times 10^{6}$ stars in the Small Magellanic Cloud (hereafter SMC) and $\sim 7 \times 10^{6}$ in the Large Magellanic Cloud (hereafter LMC). On the theoretical side, we estimate the number of stars from evolutionary model sequences computed with different amounts of overshooting. From these data sets and using evolutionary models with intermediate and low metallicity, we estimate the overshooting value during the main sequence in the SMC and LMC for a stellar mass in the range $2.5 M_{\odot}-25 M_{\odot}$.

In Sect. 2 we describe the observational data involved in this work. Section 3 is devoted to the method used: data selection and star counting. Section 4 gives the main features of our population synthesis procedure. Section 5 is devoted to astrophysical inputs, and Sect. 6 to results and effects of uncertainties. Section 7 discusses the results. It must be emphasized that we determine in fact the extent of the inner mixed core region which can be due either to true overshooting or to another process such as rotation; some observational evidence exists about correlation between metallicity and $v \sin i$ (see Venn et al. 1999). The problem of rotation is briefly discussed in Sect. 7. Finally, Sect. 8 gives some comments and concluding remarks. An appendix has been added to provide details about the population synthesis algorithm and error simulations.

\section{Observational data}

The observational data set considered here has been obtained by the Optical Gravitational Lensing Experiment (OGLE hereafter) consortium during its second operating phase 
(for more details and references the reader can consult URL: http://www . astrouw. edu.pl/ogle/).

\subsection{SMC and LMC data}

We have downloaded the SMC data described in Udalski et al. (1998). The data used in this paper are from the post-Apr. 8, 2000 revision. The SMC is divided into 11 fields (labeled SC1 to SC11) covering $55^{\prime} \times 14^{\prime}$; each field contains between $\sim 100000$ and $\sim 350000$ objects. For each object several quantities are available: equatorial coordinates, $B V I$ photometry and associated standard errors $\sigma_{B}, \sigma_{V}$ and $\sigma_{I}$. This database has the great advantage of being extensive and very homogeneous.

The LMC data are described in Udalski et al. (2000). The $B V I$ map of the LMC is composed of 26 fields (SC1 to SC26) in the central bar of the LMC. The dataset includes photometry and astrometry for about 7 million stars over a 5.7 square degree field.

\section{The star-count method}

\subsection{Data selection}

As shown in Fig. 1b, the standard error on $V$-magnitude, $\sigma_{V}$, increases with the magnitude. This is also true for $B$ or $I$ magnitudes. Hence the errors on $(B-V)$ or $(V-I)$ colors rapidly increase and reach values as large as 0.2 mag around a $V$-mag $\sim 20$ : this is of the same order as the Main Sequence width.

As we are interested in the MS structure and as we must minimize error effects while keeping quite good statistics, we have chosen to take into account only data with $\sigma_{V}$ and $\sigma_{B}$ ( or $\sigma_{I}$ ) lower or equal to $0.015 \mathrm{mag}$, leading to a maximum error on color of $0.02 \mathrm{mag}$. The value of $0.015 \mathrm{mag}$ appears to be an optimal choice maintaining a good statistics with photometric errors remaining small compared with the MS width. Figure 1 sketches the proposed selection process and displays differences between the entire Color-Magnitude Diagram (Fig. 1a) and the final diagram (Fig. 1c): obviously, the remaining data are those corresponding to lower magnitudes.

This selection process leaves $\sim 4700$ objects on the SMC MS (over a total of more than 2.2 millions objects) in the $B V$ system $(\sim 1100$ objects in the VI system $)$ and $\sim 4000$ objects on the LMC MS (over a total of more than 7.2 millions objects) in the $B V$ system ( 1600 objects in the VI system). As we can see, the $B V$ system presents more favorable statistics, therefore in the following we will work only with this set of bands.

Tables 4 from Udalski et al. (1998, Udalski et al. 2000) indicate that completeness for $V \lessgtr 18$ should be better than about 99\% for the SMC; and should be around 96\%-99\% depending on the field crowding for the LMC.

\subsection{Star count ratios: An observational constraint}

As the absolute number of stars arriving on the ZAMS per unit of time for a given mass is unknown, we rather compute star count ratios. To count stars, we first define an area
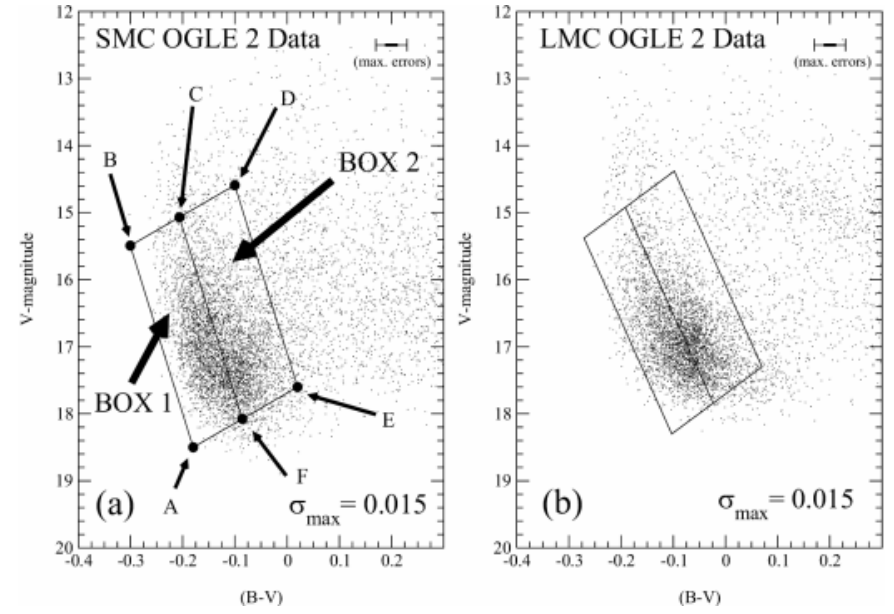

Fig. 2. a) Data from SMC with $\sigma_{V} \leq 0.015$ mag and box definitions $\left(N_{1}+N_{2}=4653\right.$ and $\left.N_{2} / N_{1}=1.08\right)$, b) the same for the LMC case $\left(N_{1}+N_{2}=4113\right.$ and $\left.N_{2} / N_{1}=1.01\right)$.

in the CM-diagram. As we are interested in the MS structure, we choose a region which contains the main sequence "bulge" revealed after the data selection process (see Fig. 2a) with the most convenient geometrical shape: a "parallelogram" (for automatic count purpose). A couple of opposite sides (AB and DE in Fig. 2a) are chosen to be more or less "parallel" to the main sequence axis.

In the CM-Diagram, main sequence stars evolve from the blue to the red side. The MS width is mainly an evolutionary effect connected to a characteristic time scale $\tau_{\mathrm{MS}}$ (time spent by a star on the Main Sequence). The distribution of the objects within the Main Sequence should be related to this time scale. Therefore we divide our parallelogram into two regions called "box 1" and "box 2" (see Fig. 2a) where the respective numbers of objects $N_{1}$ and $N_{2}$ are similar $\left(N_{2} / N_{1} \sim 1\right)$. This ratio is taken as an observational constraint and it will enable us to discriminate between theoretical grids of evolutionary tracks computed with various overshooting amounts.

We now turn to the method used to build a synthetic stellar sample comparable to the OGLE 2 ones (after selection) from evolution simulation outputs.

\section{Population synthesis}

\subsection{Evolutionary models}

Our evolutionary models are built with the 1D Henyey type code CESAM $^{1}$ (see Morel 1997) in which we brought several improvements. Applying modern techniques like the projection of the solutions on B-spline basis and automatic mesh refinements, CESAM allows robust, stable and highly accurate calculations. We use as physical inputs:

- the OPAL 96 opacities from Iglesias \& Rogers (1996) at high temperatures $(T>10000 \mathrm{~K})$ and the Alexander \& Ferguson (1994) opacities for cooler domains. For metallicity higher than the solar one (that occurs during the He core

${ }^{1}$ CESAM: Code d'Évolution Stellaire Adaptatif et Modulaire. 
burning phase) we use elemental opacities (Los Alamos) calculated by Magee et al. (1995);

- the EFF equation of state from Eggleton et al. (1973);

- elemental abundances are from Grevesse \& Noels (1993) (the "GN93" mixture), the cosmological helium is from Izotov et al. (1997): $Y_{\mathrm{P}}=0.243$, and the helium content is scaled on the solar one following a standard heliummetallicity relation: $Y=Y_{\mathrm{P}}+Z(\Delta Y / \Delta Z)$. The calibration of a solar model in luminosity yields $\Delta Y / \Delta Z=2$ (Lebreton et al. 1999) from the calibration of the solar model radius. This value is compatible with the recent value $\Delta Y / \Delta Z=$ $2.17 \pm 0.40$ of Peimbert et al. (2000). We therefore adopt $\Delta Y / \Delta Z \approx 2$;

- for the chemical composition we adopt $[\mathrm{Fe} / \mathrm{H}]$ derived from Cepheid measurements by Luck et al. (1998):

- for the SMC they find a range from -0.84 to -0.65 with a mean value: $[\mathrm{Fe} / \mathrm{H}]=-0.68$ which leads to $X_{0}=$ $0.745, Y_{0}=0.251$ and $Z_{0}=0.004$.

- for the LMC they find a range from -0.55 to -0.19 , combining all the values we obtain a mean value of $[\mathrm{Fe} / \mathrm{H}]=-0.34$ leading to: $X_{0}=0.733, Y_{0}=0.259$ and $Z_{0}=0.008$;

- The nuclear reaction rates are from Caughlan \& Fowler (1988), except: ${ }^{12} \mathrm{C}(\alpha, \gamma){ }^{16} \mathrm{O},{ }^{17} \mathrm{O}(p, \gamma){ }^{18} \mathrm{~F}$ taken from Caughlan et al. (1985) and ${ }^{17} \mathrm{O}(p, \alpha){ }^{14} \mathrm{~N}$ taken from Landré et al. (1990). The adopted rate for ${ }^{12} \mathrm{C}(\alpha, \gamma){ }^{16} \mathrm{O}$ is quite similar to the NACRE compilation (Angulo et al. 1999) one: a factor of about two higher than Caughlan \& Fowler (1988) and about $80 \%$ of Caughlan et al.'s (1985) one.

- To take into account the metallicity effect on the mass loss rate (de Jager et al. 1988) we adopt the scaling factor $\left(Z_{0} / 0.02\right)^{0.5}$ derived from the Kudritzki \& Hummer (1986) models;

- The convective flux is computed according to the classical MLT. We use a mixing length value $l_{\mathrm{MLT}}=1.6 \mathrm{H}_{\mathrm{P}}$. This value has been derived by Schaller et al. (1992) from the average location of the red giant branch of more than 75 clusters. A very similar value (1.64) has been found more recently by Lebreton et al. (1999). An extra-mixing zone is added above the Schwarzschild convective core: this "extra-mixing" zone is set to extend over the distance $l_{\text {over }}=\alpha_{\text {over }} \mathrm{H}_{\mathrm{P}}, \alpha_{\text {over }}$ being a free parameter, the value of which is discussed here;

- the external boundary conditions are determined in a layer within a simple grey model atmosphere built with an Eddington's $T(\tau)$ law.

\subsection{Conversion of the theoretical quantities into observational ones}

In order to compare theoretical results to observational data, conversions are needed. Transformations of the theoretical quantities, $\left(M_{\mathrm{bol}}, T_{\mathrm{eff}}\right)$ into absolute magnitudes and colors are derived from the most recent version of the Basel Stellar Library ( $\mathrm{BaSe} L$, version 2.2), available electronically at ftp://tangerine.astro.mat.uc.pt/pub/BaSeL/. This library provides color-calibrated theoretical flux distributions for a large range of fundamental stellar parameters, $T_{\text {eff }}(2000$ to $50000 \mathrm{~K}), \log g(-1.0$ to $5.5 \mathrm{dex})$, and $[\mathrm{Fe} / \mathrm{H}](-5.0$ to +1.0 dex). The $B \mathrm{aSe} L$ flux distributions are calibrated on the stellar $U B V R I J H K L$ colors, using:

- empirical photometric calibrations for solar metallicity;

- semi-empirical relations constructed from the color differences predicted by stellar model atmospheres for non-solar metallicities.

Details about the calibration procedure are given in Lejeune et al. (1997) and Lejeune et al. (1998). Compared to the previous versions of the $B \mathrm{aSe} L$ library, all the model spectra of stars with $T_{\text {eff }} \geq 10000 \mathrm{~K}$ are now calibrated on empirical colors from the $T_{\text {eff }}$ versus $(B-V)$ relation of Flower (1996). In addition, the calibration procedure for the cool giant model spectra has been extended in the present models to the parameter ranges $2500 \mathrm{~K} \leq T_{\text {eff }}<6000 \mathrm{~K}$ and $-1.0 \leq \log g<3.5^{2}$.

\subsection{Population synthesis}

In contrast with "classical" works on population synthesis where the CMD as a whole is simulated, we construct a small part of the CMD: the area containing the brighter MS stars. In this way the task is simplified. Artificial stellar samples have been generated from our evolutionary tracks with a specially designed population synthesis code $\mathrm{CReSyPS}^{3}$.

In our framework the main hypothesis is that the Star Formation Rate (SFR) is constant during the time scales involved here: i.e. a few hundred megayears. So for a given mass the number of observed stars (i.e. those corresponding to a given evolutionary track) must be proportional to the time scale of the main sequence. We assume that the SFR is constant in time and mass (equal for all masses in the range explored in this work), if we note $r$ the SFR: $\Delta t \approx 1 / r$ represents the mean time elapsed between two consecutive star births. For the observational star samples, $\Delta t$ is unknown but the objects numbers are available. We choose $\Delta t$ to get similar total star numbers in boxes 1 and 2 (i.e. $N_{1}+N_{2}$ ) both in the synthetic CMD and observational diagram. We point out that the ratios $N_{2} / N_{1}$ are not sensitive to the $\Delta t$ value chosen.

The evolutionary track grids scan a mass range between $2.5 M_{\odot}$ and $25 M_{\odot}$ from the ZAMS to $\log T_{\text {eff }} \sim 3.8$ covering the entire box ranges in color and magnitude (defined in Sect. 3.2). The mass step is increasing from $0.5 M_{\odot}$ around $3 M_{\odot}$ stars to $5 M_{\odot}$ above $15 M_{\odot}$. Several overshooting amounts have been used from 0.0 to 0.8 . CReSyPS treats the photometric errors by simulating OGLE 2 ones (see Appendix A) which is very important for our purposes. Our algorithm requires the knowledge of some input parameters: distance modulus, reddening and absorption, binarity rate, Initial Mass Function (hereafter IMF) slope and photometric errors.

\footnotetext{
${ }^{2}$ In the previous versions of the $B \mathrm{aSe} L$ models, we adopted $T_{\text {eff }}=$ $5000 \mathrm{~K}$ and $\log g=2.5$ as the upper limits for the calibration of giants (see Lejeune et al. 1998).

3 Code Rennais de Synthèse de Populations Stellaires.
} 
We summarize here the main steps of the algorithm:

- STEP 1: a mass distribution is generated between $2.5 M_{\odot}$ and $25 M_{\odot}$ following the Salpeter's law: $\mathrm{d} N / \mathrm{d} m \approx m^{-\alpha_{\text {Salp }}}$ (see Sect. 5.4).

- STEP 2: for each mass, an evolutionary track is interpolated within the grid calculated by the evolutionary code. On each track, models are selected every time step $\Delta t$, which is adjusted in order to yield a total number of stars equivalent to the observed one.

- STEP 3: consistently with the value of the binary rate $\langle\beta\rangle$ (see Sect. 5.3), objects are randomly selected to belong to a binary system and the magnitudes of these systems are calculated. Triple systems (and higher multiplicity systems) are neglected.

- STEP 4: distance modulus is added (and in the case of SMC a random "depth" inside the cloud) and synthetic photometric errors are attributed to magnitudes (see Appendix A).

- STEP 5: we use a "quality filter": objects with too large photometric errors are rejected from the synthetic sample.

- STEP 6: color is calculated, reddening and extinction coefficient are applied. Concerning reddening, a gaussian distribution is applied around the mean value in order to simulate object-to-object variations (see discussion in Sect. 5.2).

With the interpolation between evolutionary tracks, it is very important (particularly at low mass, i.e. 3.0 $M_{\odot} \lesssim M \lesssim 4.0 M_{\odot}$ ) to reproduce the time scale $\tau_{\mathrm{MS}}$ with a good accuracy. A test at $3.25 M_{\odot}$ has shown that the "interpolated time scale", $\tau_{\mathrm{MS}}^{\text {interpol }}$, is very close to the calculated one (with the evolutionary code) $\tau_{\text {MS }}^{\text {cal }}$ with a difference not larger than about $1 \%$. Also important are the magnitude interpolations on the Main Sequence: our tests also show a very good agreement between interpolated magnitudes and calculated ones, differences are unsignificant (about $10^{-3}-10^{-2} \mathrm{mag}$, whereas the photometric errors are much larger).

Our code intensively uses a random number generator. We have chosen an algorithm insuring a very large period about $2 \times 10^{18}$ (program "ran2" from Press et al. 1992), which is much larger than the number of synthetized objects.

As a result, examples of synthetic samples generated by CReSyPS are displayed in Fig. 3 where the influence of overshooting is shown for both clouds.

\section{Astrophysical inputs}

\subsection{Distance modulus}

Large Magellanic Cloud. The LMC distance modulus has a key role in extragalactical distance determinations, but its value is still debated. The determinations range between "short" distance scales (i.e. Stanek et al. 1998) and "long" distance scales (i.e. Laney \& Stobie 1994). Using the HIPPARCOS calibrated red clump stars, Stanek et al. (1998) found $\mu_{0, \mathrm{LMC}}=18.065 \pm$ $0.031 \pm 0.09 \mathrm{mag}$ and Laney \& Stobie (1994) from a study of Cepheids Period-Luminosity relation obtained $\mu_{0, \mathrm{LMC}}=$ 18.53 mag with an internal error of 0.04 mag. Groenewegen $\&$ Salaris (2001) found $\mu_{0, \mathrm{HV}} 2274=18.46 \pm 0.06 \mathrm{mag}$ from a study of the LMC-eclipsing binary system HV 2274. They indicate a LMC center distance at $\mu_{0, \mathrm{LMC}}=18.42 \pm 0.07 \mathrm{mag}$. Recently, from the DENIS survey data, Cioni et al. (2000) derived a distance modulus for the $\mathrm{LMC}$ of $\mu_{0, \mathrm{LMC}}=18.55 \pm 0.04$ (formal) \pm 0.08 (systematic) using a method based on the apparent magnitude of the tip of the red giant branch. The HST Key Project Team adopted $\mu_{0, \text { LMC }}=18.50 \pm 0.15$ mag (Mould et al. 2000). In order to bracket the most recent estimations, we have chosen the HST Key Project value:

$\mu_{\mathrm{LMC}}=18.50 \pm 0.15 \mathrm{mag}$.

Van der Marel \& Cioni (2001) give an order of magnitude of the depth of the LMC. They indicate small corrections to magnitude for well studied individual objects within the LMC, ranging between $\Delta \mu_{0, \mathrm{LMC}}=-0.013(\mathrm{SN} 1987 \mathrm{~A})$ to $\Delta \mu_{0, \mathrm{LMC}}=$ +0.015 (HV 2274). We neglect these corrections which have the same order of magnitude than the photometric errors.

Small Magellanic Cloud. Laney \& Stobie (1994) suggest a distance modulus (based on Cepheids) of $\mu_{0, \mathrm{LMC}}=18.94 \mathrm{mag}$ with an internal error of 0.04 mag; this modulus decreases by about 0.04 mag if calibrators are half-weighted. More recently Kovács (2000) (with a method based on double mode Cepheids), find $\mu_{0, \mathrm{LMC}}=19.05 \pm 0.13 \mathrm{mag}$ and Cioni et al. (2000) have $\mu_{0, \text { LMC }}=18.99 \pm 0.03$ (formal) \pm 0.08 (systematic) mag. We retain the following estimation:

$\mu_{\mathrm{SMC}}=18.99 \pm 0.10 \mathrm{mag}$.

The SMC distance modulus only represents an average distance. Crowl et al. (2001) have evaluated the depth of the SMC along the line-of-sight by a study of populous clusters. They derived a depth between $\sim 6 \mathrm{kpc}$ and $\sim 12 \mathrm{kpc}$; these values lead to magnitude differences of 0.2 and 0.4 mag respectively. Previous studies, see for instance Gardiner et al. (1991), show similar results with a line-of-sight SMC depth ranging between $\sim 4-7 \mathrm{kpc}$ and $\sim 15 \mathrm{kpc}$ strongly depending on the location in the SMC.

We have chosen to model the SMC depth with a gaussian distribution of distances around $\mu_{\mathrm{SMC}}$ with a standard deviation:

$$
\sigma_{\text {SMC }}^{\text {depth }}=0.05 \mathrm{mag}
$$

which represents a total depth of $\sim 8 \mathrm{kpc}$ (about $\sim 0.3 \mathrm{mag}$ ).

\subsection{Reddening and absorption}

We have to distinguish: foreground reddening $E(B-V)_{\mathrm{MW}}$ (due to material in Milky Way) and internal reddening $E(B-V)_{\mathrm{i}}$ with an origin into the Cloud itself. These quantities are expected to change along the line-of-sight. Here we model the total reddening as $E(B-V)_{\mathrm{MW}+\mathrm{i}}=E(B-V)_{\mathrm{MW}}+E(B-V)_{\mathrm{i}}$ taking into account its non-uniformity. From the literature, we derive estimations for the mean value and the dispersion of $E(B-V)_{\mathrm{MW}+\mathrm{i}}$, object-to-object variations can then be simulated.

We now discuss reddening determinations for the SMC and LMC. From a study of spectral properties of galactic nuclei behind the Magellanic Clouds, Dutra et al. (2001) have evaluated the foreground and background reddenings for both Clouds. 

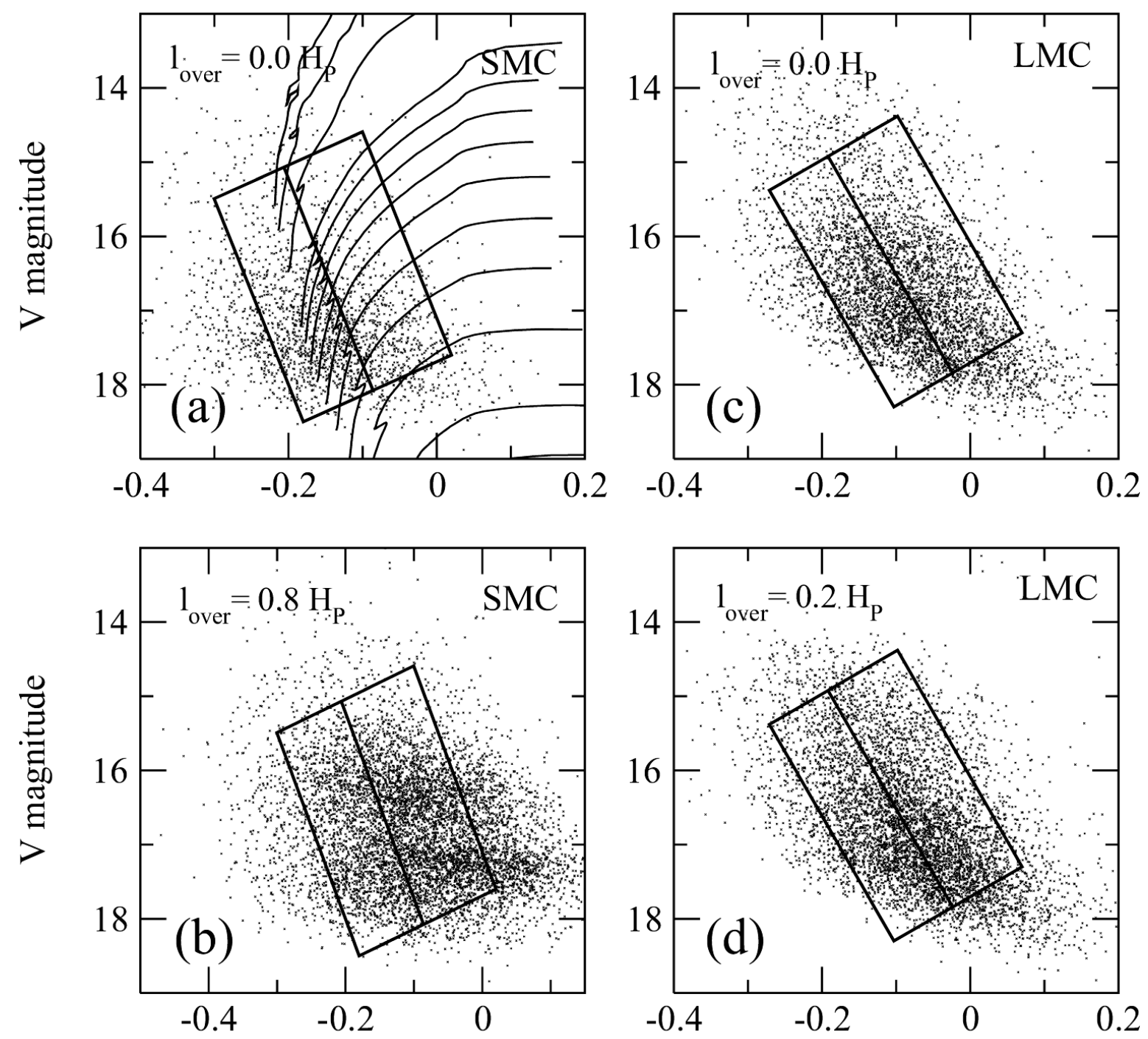

$(\mathrm{B}-\mathrm{V})$

$(\mathrm{B}-\mathrm{V})$

Fig. 3. Synthetic CM-Diagrams for SMC and LMC chemical compositions, panels a) and b) are for the SMC with two overshooting amounts: $\alpha_{\text {over }}=0.0$ and $\alpha_{\text {over }}=0.8$, panels c) and d) are for the LMC with: $\alpha_{\text {over }}=0.0$ and $\alpha_{\text {over }}=0.2$ respectively. For panel a) only $30 \%$ of the synthetic objects have been displayed for clarity purpose and corresponding evolutionary tracks have been plotted. For other panels: b)-d) the number of displayed objects has not be reduced. In all cases the total number of stars in the boxes -used for calculations- is close to the observational one: a) $N_{1}+N_{2}=4403\left(N_{2} / N_{1}=0.65\right)$, b) $N_{1}+N_{2}=5011\left(N_{2} / N_{1}=1.59\right)$, c) $N_{1}+N_{2}=3582\left(N_{2} / N_{1}=0.94\right)$, d) $N_{1}+N_{2}=4717\left(N_{2} / N_{1}=1.13\right)$. We recall that empirically we got for the SMC $N_{1}+N_{2}=4653\left(N_{2} / N_{1}=1.08\right)$ and for the LMC $N_{1}+N_{2}=4113\left(N_{2} / N_{1}=1.01\right)$. The ratios $N_{2} / N_{1}$ obtained theoretically are rather independant from the value of $N_{1}+N_{2}$ in the synthetic CM-Diagram, for instance in the case of the panel b) we got $N_{2} / N_{1}=1.60$ with $N_{1}+N_{2}=6399$. The cloud of dots in panel b) $\left(l_{\text {over }}=0.8 \mathrm{Hp}\right.$, SMC) calls a comment: it appears to be bimodal, i.e. showing over-populated regions around $V \sim 17.5 \mathrm{mag}$ and $V \sim 16.3 \mathrm{mag}$. Indeed for high overshooting values the main sequence of masses as small as $\sim 3 M_{\odot}$ can reach $V \sim 17.5$ generating with their large evolutionary time scale an over-populated region. Moreover the binarity shifts a part of this population to a $\sim 0.8$ mag brighter region, with a binarity rate $\beta=0.0$, this "bimodal effect" disappears.

For the LMC, they found an average spectroscopic reddening of $E(B-V)_{\mathrm{MW}+\mathrm{i}}=0.12 \pm 0.10 \mathrm{mag}$. The uncertainties essentially come from the determination of the stellar populations belonging to background galaxies: in the case of LMC, when Dutra et al. (2001) consider only red population galaxies, they find $E(B-V)_{\mathrm{MW}+\mathrm{i}}=0.15 \pm 0.11 \mathrm{mag}$, which gives an idea of the global uncertainty on $E(B-V)$, which should be around $\sim 0.02-0.03$ mag (about $\sim 13-20 \%$ ). For the SMC Dutra et al. (2001) find $E(B-V)_{\mathrm{MW}+\mathrm{i}}=0.05 \pm 0.05 \mathrm{mag}$. The OGLE 2 project provides reddening for each Cepheid star discovered in both Clouds. OGLE values are: $E(B-V)_{\mathrm{MW}+\mathrm{i}}=0.09 \pm 0.01$ (SMC) and $E(B-V)_{\mathrm{MW}+\mathrm{i}}=0.15 \pm 0.02$ (LMC). In Fig. 4 we have displayed the histogram of $E(B-V)_{\mathrm{MW}+\mathrm{i}}$ values from Dutra et al. (2001) and OGLE group. OGLE data have a better statistics with respectively 1333 (SMC) and 2049 (LMC) objects, against 14 (SMC) and 22 (LMC) for Dutra et al. (2001). Dutra et al's data are systematically less red; this could be inherent to their method: they observed objects behind Clouds 
and observations are easier through the more transparent regions of the clouds.

In addition, Oestreicher et al. (1995) have determined the reddening for $1503 \mathrm{LMC}$ foreground stars with a $U B V$ photometry based method: $E(B-V)_{\mathrm{MW}}=0.06 \pm 0.02 \mathrm{mag}$, a quite low value because it is related to foreground stars. It shows a spread (0.02) similar to the OGLE 2 one. Oestreicher et al.'s (1995) distribution is in very good agreement (see Fig. 4b) with Dutra et al.'s one, which tends to confirm that Dutra et al.'s result could be underestimated (Dutra et al.'s results are supposed to take account foreground and internal reddeing). Therefore in the case of LMC, we prefer to retain the OGLE average value for purpose of consistency:

$\left\langle E(B-V)_{\mathrm{MW}+\mathrm{i}}^{\mathrm{LMC}}\right\rangle=0.15 \mathrm{mag}$.

Figure $4 \mathrm{~b}$ shows that the distribution shape is the same for Dutra et al. (2001) and Oestreicher et al. (1995), the OGLE one being quite narrow which appears slightly underestimated, thus we take a value similar to Dutra et al. one:

$\sigma_{E(B-V)_{\mathrm{MW}+\mathrm{i}}}^{\mathrm{LMC}}=0.08 \mathrm{mag}$.

We take into account an additional uncertainty on $\left\langle E(B-V)_{\mathrm{MW}+\mathrm{i}}^{\mathrm{LMC}}\right\rangle$ of about:

$\delta_{E(B-V)}^{\mathrm{LMC}}=0.02 \mathrm{mag}$.

The SMC case is more questionable (we only have two sets of data), we favor the OGLE values because they are likely more suitable for performing simulations which synthesize OGLE data. Moreover OGLE data have larger statistics. We adopt:

$\left\langle E(B-V)_{\mathrm{MW}+\mathrm{i}}^{\mathrm{SMC}}\right\rangle=0.09 \mathrm{mag}$

with a crudely estimated uncertainty of about:

$\delta_{E(B-V)}^{\mathrm{SMC}}=0.015 \mathrm{mag}$.

In this case also, the OGLE standard deviation (see Fig. 4a) seems to be low, therefore we adopt the Dutra et al. (2001) one:

$\sigma_{E(B-V)_{\mathrm{MW}+\mathrm{i}}^{\mathrm{SMC}}}^{\mathrm{SM}}=0.05 \mathrm{mag}$.

The absorption coefficient is taken from Schlegel et al. (1998):

$A_{V}=3.24 \times E(B-V)_{\mathrm{MW}+\mathrm{i}}$

and is calculated for each object.

\subsection{Binary rate}

Evaluating the average binary rate $\langle\beta\rangle$ in objects as extended as the Magellanic Clouds is not easy. Locally (i.e. within a particular area of the galaxy) this multiplicity rate depends - at least - on two factors: (1) the star density and the kinematics of the objects which influence the encounter probability; (2) the initial binary rate (relative number of binaries on the ZAMS). Within the Magellanic Clouds, the binary rate likely varies over a wide range and we only consider its spatial average value $\langle\beta\rangle$.
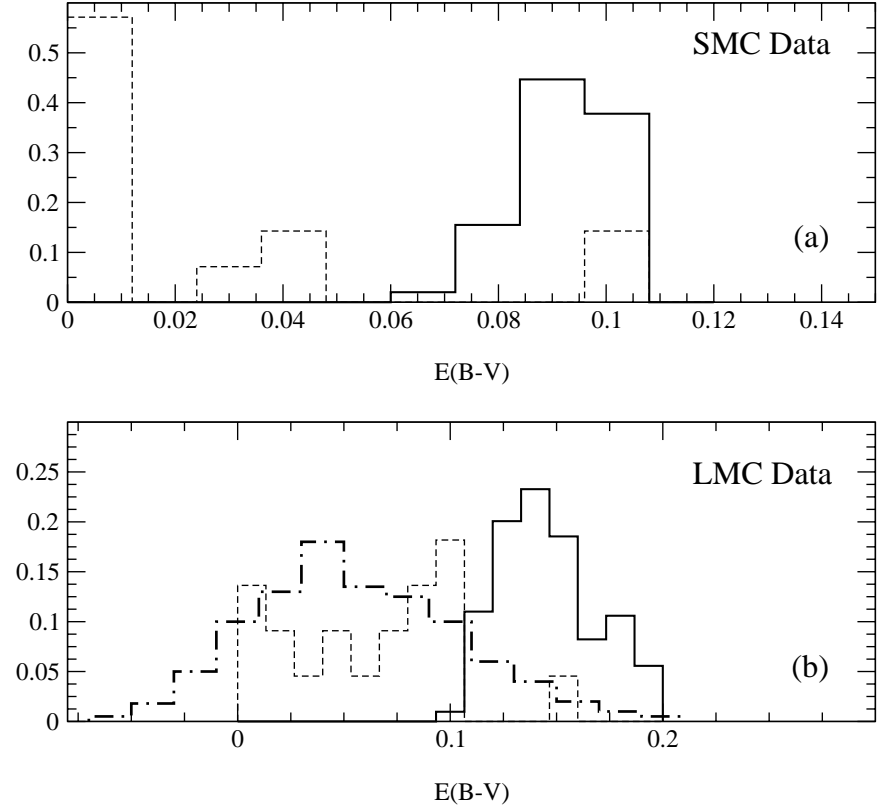

Fig. 4. Relative number of stars $N_{\text {stars }} / N_{\text {tot }}$ versus reddening from Dutra et al. (2001) (dashed curve), OGLE 2 experiment Cepheid catalogue: see Udalski et al. (1999) and Udalski et al. (1999), and data read in Fig. 24 of Oestreicher et al. (1995) (dot-dashed curve). a) Data for SMC, b) data for LMC.

Ghez (1995) finds in the solar neighbourhood that for main sequence stars and young stars the binary rate $<\beta>$ ranges between 0.10 and 0.50 (it peaks at $\langle\beta\rangle=0.50$ ). Therefore we tested the effects of binarity for these two extreme values.

In our population synthesis code, binaries are taken into account with a uniform probability for the mass ratio $q=M_{2} / M_{1}$ (in the considered mass range).

\subsection{IMF slope}

The IMF has been extensively discussed by many authors. Toward both Galactic poles and within a distance of $5.2 \mathrm{pc}$ from the Sun, Kroupa et al. (1993) found a mass function: $\mathrm{d} N / \mathrm{d} m \approx m^{-\alpha_{\text {Salp }}}$ with $\alpha_{\text {Salp }} \approx 2.7$ for stars more massive than $1 M_{\odot}$. In the LMC, Holtzman et al. (1997) inferred - from HST observations - a value consistent with the Salpeter (1955) one: $\alpha_{\text {Salp }} \approx 2.35$. At very low metallicity, Grillmair et al. (1998) observed the Draco Dwarf spheroidal Galaxy $([\mathrm{Fe} / \mathrm{H}] \approx-2)$ with the HST. They concluded that the Salpeter IMF slope remains valid in the Magellanic Clouds and we have chosen:

$\alpha_{\text {Salp }}=2.30 \pm 0.30$.

However we must keep in mind that some circularity in work exists when using an IMF. As described by Garcia \& Mermilliod (2001) the IMF can be derived from the observed Present Day Mass Function (PDMF) using evolutionary tracks and their corresponding time scales which depend on the adopted value for the overshooting! 


\subsection{Star formation rate}

For a given mass, the Star Formation Rate (SFR) represents the number of stars "created" per unit of time. Vallenari et al. (1996) have studied three stellar fields of the LMC and have found a time scale of about 2- 4 Gyr for the "bulk of star formation". We therefore make the reasonable assumption that the SFR remained quite constant during the short galactic period relevant for this work, i.e. for the last 300 Myr. The SFR involved here is an average value over each cloud.

\section{Resulting overshooting amounts}

\subsection{Large Magellanic Cloud}

As a first step we choose the mean values for each astrophysical input (discussed in Sect. 5), this yields for the LMC the following overshooting:

$l_{\text {over }}=0.09 \mathrm{H}_{\mathrm{p}}$

which is a rather mild amount. We examine in Fig. 5 how the $l_{\text {over-value is affected by the uncertainties on the astrophysical }}$ inputs:

- Changing the IMF slope $\alpha_{\text {Salp }}$ in the range 2.0-2.6 we obtain:

$0.02 \lesssim l_{\text {over }}^{\text {Salp }} \lesssim 0.09 \mathrm{H}_{\mathrm{p}}$

which tends to minimize the overshooting.

- Next, a test with the average binary rate $\langle\beta\rangle$ in the range $0.10-0.50$ leads to:

$0.00 \lesssim l_{\text {over }}^{<\beta>} \lesssim 0.14 \mathrm{Hp}$.

- A distance modulus value in the range $18.35 \lesssim \mu_{\text {LMC }} \lesssim$ 18.65 enables us to derive: $l_{\text {over }}=0.0 \mathrm{Hp}$ (in fact for $\mu_{\mathrm{LMC}}=18.35$ all the values for simulated $N_{2} / N_{1}$ are larger than the observed one) and $l_{\mathrm{over}}=0.21 \mathrm{Hp}$ for $\mu_{\mathrm{LMC}}=$ 18.65 .

- An average reddening between 0.13 and 0.17 leads respectively to $l_{\text {over }}=0.0 \mathrm{Hp}$ (in this case also all the values for simulated $N_{2} / N_{1}$ are larger than the observed one) and $l_{\text {over }}=0.27 \mathrm{H}$.

We stress that uncertainties on distance modulus and reddening infer the largest uncertainties on the final overshooting values. We retain for the LMC average chemical composition:

$l_{\text {over }}^{\mathrm{LMC}}=0.10_{-0.10}^{+0.17} \mathrm{H}_{\mathrm{p}}$

which indicates that a mild overshooting amount around $\sim 0.1-0.2 \mathrm{Hp}$ is needed to model LMC stars as found in the majority of determinations involving solar chemical composition objects (see Sect. 1).

\subsection{Small Magellanic Cloud}

For the SMC, using the mean value of each astrophysical inputs we obtain (see also Fig. 5):

$l_{\text {over }}=0.41 \mathrm{H}$.
- If the IMF slope varies between extreme values $(-2.0 \lesssim$ $\alpha_{\text {Salp }} \lessgtr-2.6$ ), the overshooting varies within the following boundaries:

$0.36 \lesssim l_{\text {over }}^{\mathrm{IMF}} \lesssim 0.50 \mathrm{H}_{\mathrm{p}}$

similarly, an average binary rate ranging between 0.10 and 0.50 leads to:

$0.34 \lesssim l_{\text {over }}^{<\beta>} \lesssim 0.45 \mathrm{H}_{\mathrm{p}}$

- The uncertainty on SMC distance modulus (18.89 $\mu_{\text {SMC }} \lesssim 19.09$ ) leads to:

$0.37 \lesssim l_{\text {over }}^{\mu} \lesssim 0.46 \mathrm{H}_{\mathrm{p}}$

- Similarly if one considers the uncertainty on the average reddening $(<E(B-V)>$ ranging between 0.075 and $0.105)$, the overshooting amount shows a high sensitivity to reddening:

$0.36 \lesssim l_{\text {over }}^{E(B-V)} \lesssim 0.52 \mathrm{H}_{\mathrm{p}}$.

Again, uncertainties on distance modulus and reddening are the largest. We retain for the SMC:

$l_{\text {over }}^{\text {SMC }}=0.40_{-0.04}^{+0.12} \mathrm{Hp}$.

Whatever the simulation is, statistical errors are of the order of $0.01 \mathrm{H}_{\mathrm{p}}$ which can be safely neglected. In the SMC case, the required overshooting appears to be much larger than for LMC stars and for solar composition stars.

\section{Discussion}

\subsection{An upper limit with Roxburgh's criterion}

Roxburgh's criterion (Roxburgh 1989) is a very general constraint on the size of the convective core. It is written as an integral formulation over the stellar core radius:

$\int_{r=0}^{r=R_{\text {core }}}\left(L_{\text {rad }}-L_{\text {nuc }}\right) \frac{1}{T^{2}} \frac{\mathrm{d} T}{\mathrm{~d} r} \mathrm{~d} r=\int_{r=0}^{r=R_{\text {core }}} \frac{\Phi}{T} 4 \pi r^{2} \mathrm{~d} r$

where $L_{\mathrm{rad}}$ and $L_{\mathrm{nuc}}$ are respectively the radiative energy flux and the total energy flux (in $\mathrm{J} \mathrm{s}^{-1}$ ) generated by nuclear processes, $r$ is the radius, $R_{\text {core }}$ is the core size including the "overshooting" region. $\Phi$ represents the viscous dissipation (in $\mathrm{J} \mathrm{s}^{-1} \mathrm{~m}^{-3}$ ). In the whole stellar convective core the turbulence is supposed to be statistically stationary and the temperature gradient has to be almost adiabatic. In Eq. (1) the integrand is positive when $r$ is lower than the Schwarzschild boundary where $L_{\mathrm{rad}}=L_{\text {nuc }}$ and it becomes negative beyond.

The viscous dissipation $\Phi$ is unknown but the integral constraint is satisfied for larger $R_{\text {core }}$ value when $\Phi=0$. Hence, neglecting the dissipation by setting $\Phi=0$ provides the maximum possible extent of the convective core which can be considered as the upper limit for overshooting. Evolutionary tracks have been calculated, using Roxburgh's criterion, for a representative mass of $6 M_{\odot}$ and SMC and LMC metallicities. The equivalent overshooting amount (EOA), given in Table 1 , is the time weighted average overshooting distance along the evolutionary tracks, expressed in pressure scale height. 

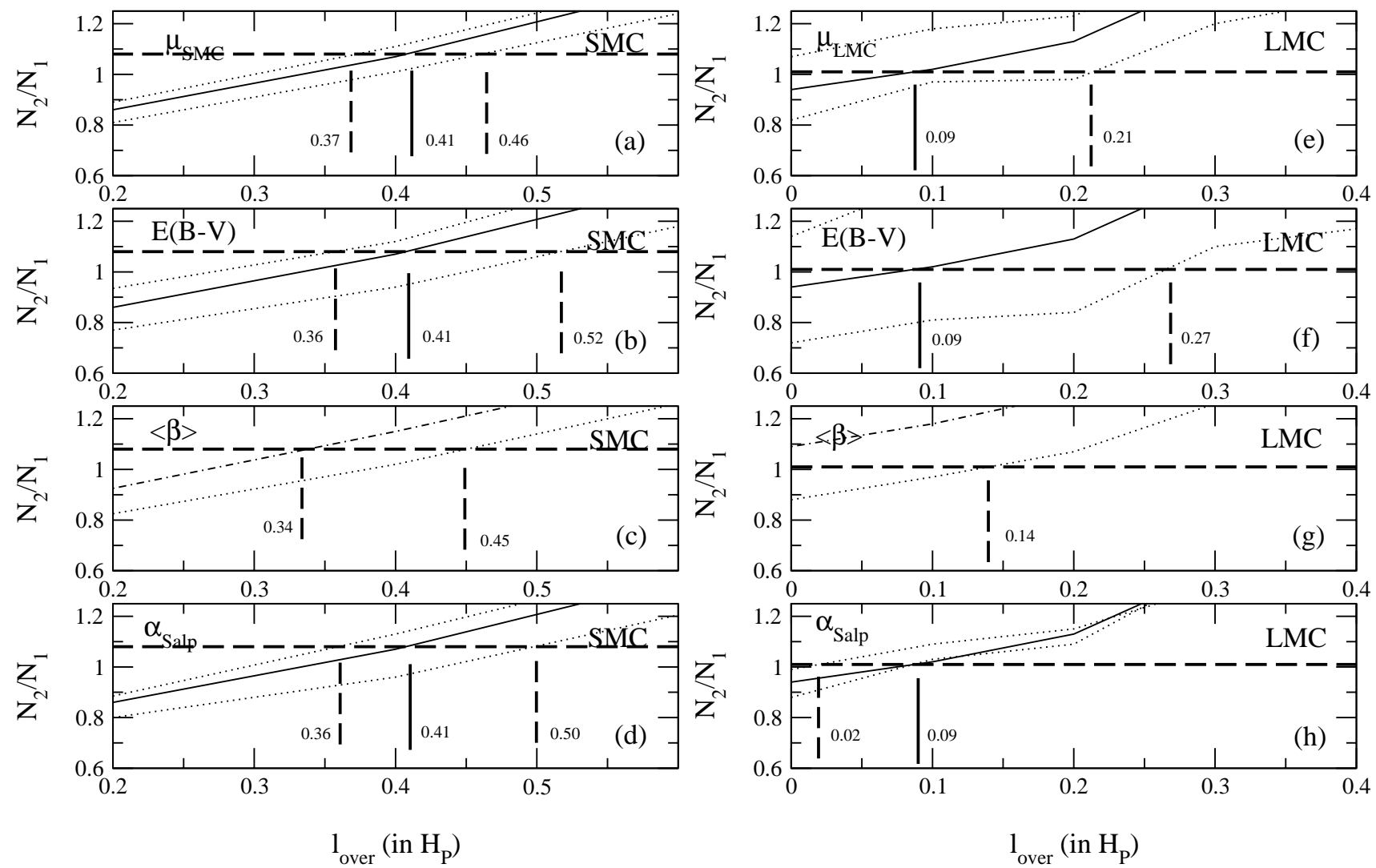

Fig. 5. Overshooting determinations for SMC (panels a-d)) and LMC (panels e-h)). The influence of distance modulus, reddening and IMF slope are considered for each cloud: continuous lines correspond to central values of these parameters discussed in Sect. 5 and dashed lines to the associated error bars. Inferred values of $l_{\text {over }}$ (and uncertainties) are given on each figure. For $\mathbf{c}$ ) and $\mathbf{g}$ ) panels, dotted line is for $<\beta>=0.0$ and dot-dashed for $\langle\beta\rangle=0.5$ (see text).

Table 1. Time weighted average overshooting distances for a $6 M_{\odot}$ main sequence model, derived with the "Roxburgh's criterion" neglecting dissipative phenomenon $(\Phi=0)$.

\begin{tabular}{ccc}
\hline \hline Metallicity $Z_{0}$ & $0.004(\mathrm{SMC})$ & $0.008(\mathrm{LMC})$ \\
Average EOA & $0.6 \mathrm{H}_{\mathrm{p}}$ & $0.6 \mathrm{H}_{\mathrm{p}}$ \\
\hline
\end{tabular}

In both cases (LMC and SMC), Roxburgh's criterion predicts a maximum value (i.e. neglecting viscous dissipation) around $0.6 \mathrm{Hp}$ (see Table 1) independent from $Z_{0}$. Our determinations - i.e. $l_{\text {over }}^{\mathrm{SMC}}=0.40_{-0.06}^{+0.12} \mathrm{H}_{\mathrm{p}}$ and $l_{\mathrm{over}}^{\mathrm{LMC}}=0.10_{-0.10}^{+0.17} \mathrm{H}_{\mathrm{p}^{-}}$ therefore are compatible with the theoretical upper limit given by the Roxburgh's criterion.

\subsection{Influence of rotation}

In addition to convection, rotation is an other important phenomenon inducing mixing through shear effects and other instabilities. For instance Venn (1999) finds surface abundance variations in SMC A supergiants that could be explained by some kind of mixing related to rotation.

Taking account of the rotational effect brings new important unknown features: (1) the $\Omega$-value distribution and (2) the $v \sin i$ distribution for the considered stellar population. Both features remain unconstrained by observational studies.
In addition, stellar rotation involves many effects and physical processes that are non-trivial to include in modern evolutionary codes. Talon et al. (1997) show that (see their Fig. 5) a rotating 1D-model with an initial surface velocity of $300 \mathrm{~km} \mathrm{~s}^{-1}$ leads to a main sequence track equivalent to an overshooting model using $l_{\text {over }}=0.2 \mathrm{Hp}$. Despite great theoretical efforts, a free parameter remains for horizontal diffusivity in Talon et al. (1997) treatment of rotational mixing (see Zahn 1992).

Rotation changes the global shape of an evolutionary track, through two distinct effects: (1) the material mixing inside the inner part of the star which brings more fuel into the nuclear burning zones like overshooting, (2) the effective surface gravity modification leading to color and magnitude changes (which depend on the angle between the line-of-sight and the rotational axis). In their Fig. 6, Maeder \& Meynet (2001) show the influence of rotation on evolutionary tracks for low metallicity objects $\left(Z_{0}=0.004\right)$. These tracks have been calculated taking into account: (1) an "average effect" on surface, (2) the internal mixing. These tracks are very similar to those calculated with different overshooting amounts values.

An additional effect which needs to be discussed here is the surface effect: modifications of colors and magnitudes of MS stars due to rotation (in absence of any mixing phenomenon) have been studied by Maeder \& Peytremann (1970) with uniformly rotating models. Their Table 2 gives expected changes of $M_{V}$ and $(B-V)$ as a function of $\Omega$ (angular velocity 


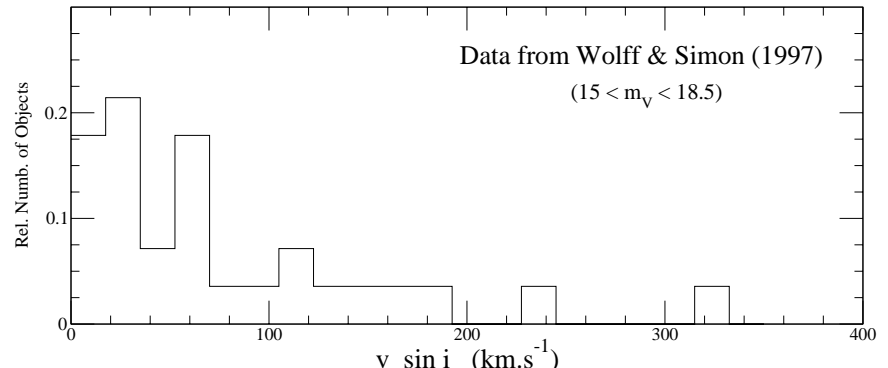

Fig. 6. Relative number of stars $\left(N_{\text {stars }} / N_{\text {tot }}\right.$ versus $V \times \sin i$ for Wolff $\&$ Simon (1997) objects with magnitudes in our studied range.

expressed in break-up velocity unit) and $v \sin i$ (this latter ranges from 0 to $457 \mathrm{~km} \mathrm{~s}^{-1}$, for a $5 M_{\odot}$ star). In this table standard deviation for $M_{V}$ and $(B-V)$, are: $\sigma_{M_{V}}=0.18 \mathrm{mag}$ and $\sigma_{(B-V)}=0.01 \mathrm{mag}$. Therefore the rotation effect has roughly the same order of magnitude than present uncertainties on magnitudes and colors. However, stars with $v \sin i$ greater than $\sim 200 \mathrm{~km} \mathrm{~s}^{-1}$ are quite rare, as shown by the data of Wolff \& Simon (1997) (see Fig. 6). Then keeping only data with $v \sin i \lesssim 200 \mathrm{~km} \mathrm{~s}^{-1}$, leads to: $\sigma_{M_{V}}=0.20 \mathrm{mag}$ and $\sigma_{(B-V)}=0.001 \mathrm{mag}$. The effect on absolute magnitude remains of the same order, whereas the effect on color becomes largely negligible. We conclude that our results remain valid, even if the major mixing is due to rotation. In this case, the value of $l_{\text {over }}$ would change its meaning. Major contribution to $l_{\text {over }}$ value would represent a shear effect mixing.

\subsection{Influence of chemical composition gradient}

We have sofar assumed a uniform chemical composition. The chemical composition may vary inside each Magellanic Cloud. The existence of an abundance gradient in the Clouds is still debated and spectroscopic measurements with a statistics as large as the statistics of OGLE 2 data are not available. In their Table 4, Luck et al. (1998) give spectroscopic determinations of $[\mathrm{Fe} / \mathrm{H}]$ for $7 \mathrm{SMC}$ Cepheids and 10 LMC Cepheids. For SMC data, the standard deviation is $\sigma_{[\mathrm{Fe} / \mathrm{H}]}^{\mathrm{SMC}} \sim 0.07$ dex leading to negligible variations for the heavy elements mass fraction $Z_{0}$. Therefore the SMC can be considered as chemically homogeneous for our purpose. For LMC, Luck et al. (1998) find a standard deviation $\sigma_{[\mathrm{Fe} / \mathrm{H}]}^{\mathrm{LMC}} \sim 0.10$ dex giving $0.007 \lessgtr Z_{0} \lesssim 0.01$. From evolutionary tracks of typical mass $\left(6 M_{\odot}\right)$ and an overshooting of $0.1 \mathrm{H}_{\mathrm{P}}$, changing $Z_{0}$ from 0.007 to 0.01 has a negligible effect on magnitude and an effect of $\sim 0.003$ mag on color, which is largely lower than the photometric errors. We conclude that - in the light of the present knowledge - the chemical composition gradient does not change our results significantly.

\subsection{Comparison with other works}

From the investigation of young clusters in the Magellanic Clouds, Keller et al. (2001) did not find any noticeable overshooting dependence with metallicity. They obtained for NGC $330\left(Z_{0} \sim 0.003\right) l_{\text {over }}^{\text {NGC } 330}=0.34 \pm 0.10 \mathrm{H}_{\mathrm{p}}$, which is compatible with our determination for the SMC: $l_{\mathrm{over}}^{\mathrm{SMC}}=$ $0.40_{-0.04}^{+0.12} \mathrm{H}_{\mathrm{p}}$. For NGC $2004\left(Z_{0} \sim 0.007\right)$ Keller et al. (2001)

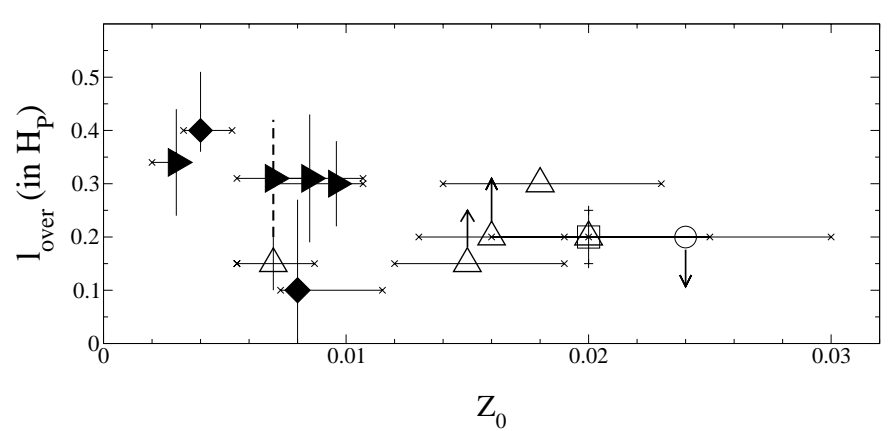

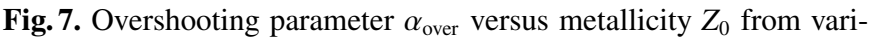
ous sources. Open triangles represent results from Ribas et al. (2000) for SZ Cen $\left(Z_{0} \sim 0.007\right)$ error bars have been indicated, arrows mean that the derived value is a minimum. The open square shows a result from Kozhurina-Platais et al. (1997) for the galactic cluster NGC 3680, error bars are indicated. Filled triangles are determinations from Keller et al. (2001): with continuous error bars amounts corresponding to the SMC cluster NGC 330, NGC 1818, NGC 2100 and with dashed error bars result for the LMC cluster NGC 2004. Open circle: determination in Hyades cluster from Lebreton et al. (2001) (upper limit for overshooting). Filled diamonds: SMC and LMC determinations performed in this work. Errors on $Z$ have been evaluated assuming an internal error on $[\mathrm{Fe} / \mathrm{H}]$ of $0.1 \mathrm{dex}$.

got $l_{\mathrm{over}}^{\mathrm{NGC} 2004}=0.31 \pm 0.11 \mathrm{H}$; while for similar metallicity we derived $l_{\text {over }}^{\mathrm{LMC}}=0.10_{-0.10}^{+0.17} \mathrm{Hp}$ which is also compatible with Keller et al.'s result. One can note that masses involved in our simulations (average mass of $\sim 7-8 M_{\odot}$ with a standard deviation of $4 M_{\odot}$ ) are higher than the Keller et al. (2001) one (terminus masses in the range $9-12 M_{\odot}$ for the four clusters). Keller et al. (2001) do not discuss the influence of the uncertainty on distance modulus of the clusters and use $\mu_{\mathrm{LMC}}=18.45 \mathrm{mag}$ and $\mu_{\mathrm{SMC}}=18.85 \mathrm{mag}$.

Ribas et al. (2000) derive overshooting amounts from evolutionary models of galactic binary systems. For SZ Cen $\left(Z_{0} \sim\right.$ 0.007 ) they find $0.1 \lessgtr l_{\text {over }} \lesssim 0.2 \mathrm{H}_{\mathrm{p}}$ which is close to our value for the LMC, but the mass of SZ Cen is $2.32 M_{\odot}$ and some mass effect cannot be avoided, therefore any comparison with the present results must be considered with care. In Fig. 7 we summarize results from several authors. Despite the small number of points, a slight dependence of overshooting with metallicity cannot be excluded. However, at low and high metallicities, the considered mass ranges are different and the errors remain substantial, therefore a definite conclusion is not yet possible.

\section{Conclusion}

In this paper we have estimated the overshooting distance from a turbulent core for intermediate-mass main sequence stars. The result for SMC is $l_{\mathrm{over}}^{\mathrm{SMC}}=0.40_{-0.06}^{+0.12} \mathrm{H}_{\mathrm{P}}$, and for the LMC $l_{\text {over }}^{\mathrm{LMC}}=0.10_{-0.10}^{+0.17} \mathrm{H}_{\mathrm{P}}$. The main contributions to errors are those brought by distance modulus and reddening uncertainties. We have shown that chemical gradients within the clouds and rotation surface effects of studied stars cannot significantly influence our results. Binary rate and IMF slope have no important effects as well. For SMC, despite different methods and data, we find a result very similar to Keller et al.'s (2001) one for 
cluster NGC 330. The case of LMC is more questionable because of the rather large uncertainty on reddening.

Figure 7 tends to indicate a sensitivity of overshooting to metallicity. However a mass effect cannot be excluded; we can only stress that if such a dependence exists, it should be an increase of overshooting with decreasing metallicity. However, the overshooting is expected to increase with mass, unfortunately samples studied at solar metallicity have often lower masses than those at low metallicities. Therefore further investigations are needed to disentangle these effects. In any cases, if this dependence is confirmed the next challenge will be the physical explanation of this metallicity-overshooting effect.

Finally, the overshooting amounts derived in this work have a statistical meaning: they are average values over time (in real stars, "overshooting" likely changes during the main sequence) and over mass in the considered range. Moreover these amounts represent an extramixing above the classical core generated either by inertial penetration of convective bubbles or shear phenomena related to rotation. The real extent of the core likely results from a combination of both processes; indeed, rotating models Maeder \& Meynet (2001)'s rotating models still need overshooting.

Acknowledgements. We thank Jean-Paul Zahn and Ian Roxburgh for helpful discussions; we are also grateful to the OGLE group for providing their data and to Pierre Morel for writing the CESAM code. We thank the referee Dr. S. C. Keller for valuable remarks and suggestions.

\section{Appendix A: Photometric error simulations}

As we selected the data using a criterion involving the photometric standard deviation of magnitude measurements, we have to generate an artificial standard deviation for the theoretical magnitude computed from evolutionary models. Moreover the general properties of the synthetic standard deviation distribution must be similar to the OGLE 2 one.

We describe here the scheme used to generate the pseudosynthetic photometric standard error distributions. The prefix "pseudo" means that we have extracted information about the standard error distribution from the OGLE 2 data themselves (see Fig. A.1a). For that purpose, we divide the relevant range of magnitudes into bins; in each bin, we construct the histogram of standard deviation values (Fig. A.1b). This histogram then is fitted with a function of the form:

$P(\sigma)=a \times\left(\sigma-\sigma_{\min }\right)^{4} \times \mathrm{e}^{-b\left(\sigma-\sigma_{\min }\right)}$

where the constants $a, b, \sigma, \sigma_{\min }$ are derived from the OGLE 2 data. $P(\sigma)$ represents the probability for having the standard deviation $\sigma$. The constants have been derived for each "magnitude bin", for each OGLE fields in SMC and LMC. Then average values have been calculated over SMC and LMC.

In our population synthesis code, for a given magnitude value $m$, a standard deviation value $\sigma_{m}$ is randomly determined following the probability law derived from OGLE. After that, either the object is rejected (if the $\sigma_{m}$ value is too large) or
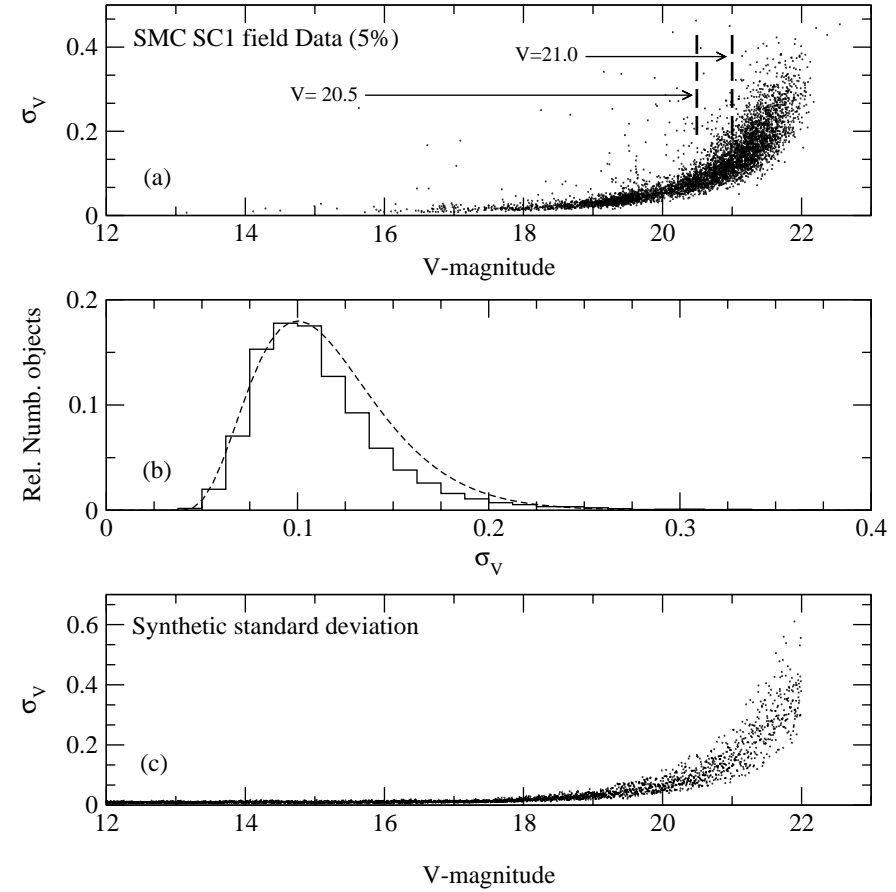

Fig. A.1. a) Standard deviation $\sigma_{V}$ versus $V$-magnitude for objects belonging to the SC1 field of the SMC. b) Histogram of the $\sigma_{V}$ values for magnitude $V$ between 20.5 and 21.0, the fit (dashed curve) is performed with a function of a type given in the text. Differences between the fit and the histogram are clearly insignificant for our purpose. c) Synthetic $\sigma_{V}$ distribution generated with our algorithm.

the magnitude $m$ is changed into $m^{\text {noisy }}$, following a gaussian distribution having a standard deviation $\sigma_{m}$.

Let us comment about differences between Figs. A.1a and A.1c. Figure A.1a contains the "evolutionary information" - i.e. more objects at high magnitudes- whereas Fig. A.1c does not contain this information, objects have been uniformly distributed with respect to the magnitude. These facts explain the difference between both figures.

\section{References}

Alexander, D., \& Ferguson, J. 1994, ApJ, 437, 879

Alongi, M., Bertelli, G., Bressan, A., \& Chiosi, C. 1991, A\&A, 244, 95

Andersen, J. 1991, A\&AR, 3, 91

Angulo, C., Arnould, M., Rayet, M., et al. 1999, Nucl. Phys. A, 656, 3

Bertelli, G., Bressan, A., Chiosi, C., Fagatto, F., \& Nasi, E. 1994, A\&AS, 106, 275

Böhm-Vitense, E. 1958, Zs. f. Ap., 46, 135

Bressan, A. G., Chiosi, C., \& Bertelli, G. 1981, A\&A, 102, 25

Caughlan, G., \& Fowler, W. 1988, Atomic Data Nuc. Data Tables, 40, 283

Caughlan, G., Fowler, W., Harris, M., \& Zimmerman, B. 1985, Atomic Data Nuc. Data Tables, 32, 197

Charbonnel, C., Meynet, G., Maeder, A., \& Schaerer, D. 1996, A\&AS, 115,339

Cioni, M.-R. L., Van der Marel, R. P., Loup, C., \& Habing, H. J. 2000, A\&A, 359, 601

Crowl, H. H., Sarajedini, A., Piatti, A., et al. 2001, AJ, 122, 220 
de Jager, C., Nieuwenhuijzen, H., \& van der Hucht, K. A. 1988, A\&AS, 72, 259

Dutra, C. M., Bica, E., Clariá, J. J., Piatti, A. E., \& Ahumada, A. V. 2001, A\&A, 371, 895

Eggleton, P., Faulkner, J., \& Flannery, B. 1973, A\&A, 23, 325

Flower, P. J. 1996, ApJ, 469, 355

Garcia, B., \& Mermilliod, J. C. 2001, A\&A, 368, 122

Gardiner, L. T., Hatzidimitriou, D., \& Hawkins, M. R. S. 1991, Proc. Astron. Soc. Austr., 9, 80

Ghez, A. 1996, in Evolutionary processes in Binary Stars, ed. R. A. M. J. Wijers, M. B. Davis, \& C. A. Tout (Kluwer Academic Publishers), 1

Grevesse, N., \& Noels, A. 1993, in Origin and Evolution of the Elements, ed. V.-F. E. Prantzos N., \& C. M. (Cambridge University Press, Cambrigge), 14 (GN'93)

Grillmair Truc, T., \& Truc, T. 1998, AJ, 115, 144

Groenewegen, M. A. T., \& Salaris, M. 2001, A\&A, 366, 752

Holtzman, Truc, T., \& Truc, T. 1997, AJ, 113, 656

Iglesias, C. A., \& Rogers, F. J. 1996, ApJ, 464, 943 (OPAL 96)

Iwamoto, N., \& Saio, H. 1999, ApJ, 521, 297

Izotov, Y., Thuan, T., \& Lipovetsky, V. 1997, ApJS, 108, 1

Keller, S. C., Da Costa, G. S., \& Bessell, M. S. 2001, AJ, 121, 905

Kovács, G. 2000, A\&A, 363, L1

Kozhurina-Platais, V., Demarque, P., Platais, I., Orosz, J. A., \& Barnes, S. 1997, AJ, 113, 1045

Kroupa, P., Tout, C., \& Gilmore, G. 1993, MNRAS, 262, 545

Kudritzki, R., \& Hummer, D. 1986, in IAU Symp. 116, Luminous Stars and Associations in Galaxies, ed. P. L. C. de Loore, \& A. J. Willis, 3

Landré, V., Prantzos, N., Aguer, P., et al. 1990, A\&A, 240, 85

Laney, C. D., \& Stobie, R. S. 1994, MNRAS, 266, 441

Lebreton, Y., Fernandes, J., \& Lejeune, T. 2001, A\&A, 374, 540

Lebreton, Y., Perrin, M.-N., Cayrel, R., Baglin, A., \& Fernandes, J. 1999, A\&A, 350, 587

Lejeune, T., Cuisinier, F., \& Buser, R. 1997, A\&AS, 125, 229

Lejeune, T., Cuisinier, F., \& Buser, R. 1998, A\&AS, 130, 65

Luck, R. E., Moffett, T. J., Barnes, T. G., \& Gieren, W. P. 1998, AJ, 115,605

Maeder, A., \& Mermilliod, J. 1981, A\&A, 93, 136

Maeder, A., \& Meynet, G. 2001, A\&A, 373, 555

Maeder, A., \& Peytremann, E. 1970, A\&A, 7, 120
Magee, N. H., Abdallah, J. Jr., Clark, R. E. H., et al. 1995, in Atomic Structure Calculations and New Los Alamos Astrophysical Opacities, ed. S. Adelman, \& W. Wiese, vol. 78 (Astronomical Society of the Pacific Conf. Ser. (Astrophysical Applications of Powerful New Database)), 51

Massaguer, J. 1990, in Rotation and Mixing in Stellar Interiors, ed. M.-J. Goupil, \& J.-P. Zahn (Springer, Berlin, Heidelberg, New York), 129

Mermilliod, J.-C., \& Maeder, A. 1986, A\&A, 158, 45

Morel, P. 1997, A\&AS, 124, 597

Mould, J. R., Huchra, J. P., Freedman, W. L., et al. 2000, ApJ, 529, 786

Oestreicher, M. O., Gochermann, J., \& Schmidt-Kaler, T. 1995 , A\&AS, 112, 495

Peimbert, M., Peimbert, A., \& Ruiz, M. 2000, ApJ, 541, 688

Press, W., Teukolsky, S., Vetterling, W., \& Flannery, B. 1992, Numerical Recipes in Fortran 77 (Cambridge University Press)

Ribas, I., Jordi, C., \& Giménez, Á. 2000, MNRAS, 318, L55

Rogers, F. J., \& Iglesias, C. A. 1992, ApJS, 79, 507

Roxburgh, I. 1989, A\&A, 211, 361

Salpeter, E. 1955, ApJ, 121, $161 \mathrm{~S}$

Schaller, G., Schaerer, D., Meynet, G., \& Maeder, A. 1992, A\&AS, 96, 269

Schlegel, D. J., Finkbeiner, D. P., \& Davis, M. 1998, ApJ, 500, 525

Schröder, K., Pols, O. R., \& Eggleton, P. P. 1997, MNRAS, 285, 696

Stanek, K. Z., Zaritsky, D., \& Harris, J. 1998, ApJ, 500, L141

Stothers, R. B., \& Chin, C. 1991, ApJ, 381, L67

Talon, S., Zahn, J.-P., Maeder, A., \& Meynet, G. 1997, A\&A, 322, 209

Udalski, A., Soszynski, I., Szymanski, M., et al. 1999a, Acta Astron., 49, 223

Udalski, A., Soszynski, I., Szymanski, M., et al. 1999b, Acta Astron., 49,437

Udalski, A., Szymański, M., Kubiak, M., et al. 1998, AcA, 48, 147

Udalski, A., Szymański, M., Kubiak, M., et al. 2000, AcA, 50, 307

Vallenari, A., Chiosi, C., Bertelli, G., Aparicio, A., \& Ortolani, S. 1996, A\&A, 309, 367

Van der Marel, R. P., \& Cioni, M. L. 2001, AJ, 122, 1807

Venn, K. 1999, ApJ, 518, 405

Wolff, S., \& Simon, T. 1997, PASP, 109, 759

Zahn, J. 1991, A\&A, 252, 179

Zahn, J.-P. 1992, A\&A, 265, 115 